TRANSACTIONS OF THE

AMERICAN MATHEMATICAL SOCIETY

Volume 319, Number 1, May 1990

\title{
A COMPLETE CLASSIFICATION OF THE PIECEWISE MONOTONE FUNCTIONS ON THE INTERVAL
}

\author{
STEWART BALDWIN
}

\begin{abstract}
We define two functions $f$ and $g$ on the unit interval $[0,1]$ to be strongly conjugate iff there is an order-preserving homeomorphism $h$ of $[0,1]$ such that $g=h^{-1} f h$ (a minor variation of the more common term "conjugate", in which $h$ need not be order-preserving). We provide a complete set of invariants for each continuous (strictly) piecewise monotone function such that two such functions have the same invariants if and only if they are strongly conjugate, thus providing a complete classification of all such strong conjugacy classes. In addition, we provide a criterion which decides whether or not a potential invariant is actually realized by some piecewise monotone continuous function.
\end{abstract}

\section{INTRODUCTION}

1. Definition. Let $I$ be the unit interval. If $f, g$ are maps on $I$ then $f$ and $g$ are conjugate iff there is a homeomorphism $h$ of $I$ such that $f=$ $h^{-1} g h$. A more convenient term for our purposes will be: $f, g$ are strongly conjugate (written $f \sim g$ ) iff there is an order preserving homeomorphism $h$ as above. Given a map $f$ on $I$, define the map $f^{*}$ by $f^{*}(x)=1-$ $f(1-x)$ (essentially a $180^{\circ}$ rotation of the graph). The main result of this paper will be to give a complete set of invariants for the strong conjugacy classes of the piecewise monotone continuous functions on the unit interval $I=[0,1]$. Requiring the homeomorphisms $h$ above to be order-preserving will simplify all of the arguments considerably. However, since $f$ and $g$ are conjugate iff $f \sim g$ or $f^{*} \sim g$, a classification of the $\sim$-classes will yield a classification of the conjugacy classes.

What do we mean by a "classification"? Essentially we want to assign to each piecewise monotone function $f$ a complete set of invariants, or "classification", $C_{f}$ such that $f \sim g$ iff $C_{f}=C_{g}$. A few of the properties that something called

Received by the editors September 4, 1987 and, in revised form, July 26, 1988. This paper was presented at the 1987 Spring Topology Conference at the University of Alabama at Birmingham, Birmingham, Alabama.

1980 Mathematics Subject Classification (1985 Revision). Primary 58F08; Secondary 58F13.

Key words and phrases. Topologically conjugate, kneading sequence. 
a classification ought to have are:

(1) Use of equivalence classes should be avoided as much as possible (for example, defining $C_{f}$ to be the $\sim$-equivalence class of $f$ is obviously cheating). While it will not be possible to avoid them completely, there will be a large subclass of the piecewise monotone functions for which they are unnecessary.

(2) $C_{f}$ should provide information about the behavior of the function $f$. In our case we will be getting substantial information about the behavior of the dynamical system defined by the function $f$.

(3) Since there are continuum many -classes it is impossible to make any such classification "effective" in the most desirable sense of the word, but there should be effective algorithms which work on a reasonably large subclass of equivalence classes.

Once a complete set of invariants has been found for some problem, a natural question arises which is basically the converse of the classification. That is, given an item $C$ which "looks like" it is the invariant arising from some function $f$, is there a "reasonable" way of deciding whether or not a function $j$ exists such that $C_{f}=C$ (where, of course, the criterion "such an $f$ exists" is not considered "reasonable"). Thus, an additional property which is desirable is:

(4) There should be a rule in the terminology of the classification $C_{f}$ (i.e. not referring to the functions themselves) which decides whether or not a given potential $C_{f}$ is actually realized by some function $f$.

This paper will first consider the classification problem, and Theorem 18 below will give us (1), (2), and (3). Then, the abstract machinery needed for the "realization" problem will be introduced, culminating in Theorem 54, the solution to (4) above.

There have been a number of results which give a classification of some subset of the piecewise monotone functions on the interval. In [P], William Parry showed that if in addition $f$ is strongly transitive (i.e. for every nonempty open subset $U$ of $I$ and every $x$ in $I$, there is an integer $n$ and a $y$ in $U$ such that $f^{n}(y)=x$ ) then $f$ is conjugate to a piecewise linear map $g$ such that for some fixed constant $c>1, g^{\prime}(x)$ is $c$ or $-c$ on all monotone intervals. This function $g$ is unique up to scale. For the case of unimodal functions, John Guckenheimer provided a classification of all such functions having negative Schwartzian derivative (see $[\mathrm{G}, \mathrm{CE}]$ ). In $[\mathrm{BC}]$, Louis Block and Ethan Coven gave a classification of a subclass of the piecewise monotone functions which had the additional advantage of being "effective" in the following very strong sense: each such function was conjugate to a function that could be described with a finite amount of information, and there was an effective algorithm which decided whether or not any two such descriptions resulted in conjugate functions. The class of functions considered in that result was the set of all continuous functions which were either expanding piecewise monotone with all orbits of extrema finite or piecewise linear with all orbits of endpoints of piecewise linear pieces being finite. 
One of the most useful ideas in dealing with functions on $I$ has been the concept of kneading sequences, or itineraries. For the case of unimodal maps the main idea seems to have first appeared in a paper by Metropolis, Stein, and Stein [MSS], and the more general case for piecewise monotone functions was first treated in detail by Milnor and Thurston [MT]. That itineraries tell the whole story (as far as conjugacy class) for the expanding piecewise monotone functions has been known for several years, but the details do not seem to have ever appeared in print. I am not sure who first noticed it, but it was a natural observation once the idea of itineraries appeared, and it may be that it was noticed independently by several mathematicians. The proof of this fact appears below as Theorem 11. The main difficulty lies in dealing with functions which are not expanding, especially when there are many attracting periodic orbits of different periods, something that cannot happen with expanding maps. To get the general result it is necessary to find more invariants which, when added to the information about itineraries, will give a complete classification of the continuous piecewise-monotone functions on the interval. Before we can do that, some preliminaries are necessary.

2. Definition. A function $f$ on an interval of reals is called piecewise monotone (abbreviated pwm hereafter) iff $f$ is continuous and there are a finite number of subintervals $J_{1}, J_{2}, J_{3}, \ldots, J_{n-1}, J_{n}$ covering the interval such that $f$ is either strictly increasing or strictly decreasing on each $J_{i}$. Note that we are not allowing a pwm function to be constant on any nontrivial interval. Let $f$ be a pwm function on the unit interval (or, more generally, any bounded closed interval of reals), and let $N=N_{f}$ be the number of maximal monotone intervals of $f$. For each even $k, 0<k<2 N$, let $t_{k}=t_{k}(f)$ be defined so that $0=t_{0}<t_{2}<t_{4} \cdots<t_{2 N}=1$ and each $t_{k}$ is a relative minimum or maximum of $f$. Define intervals $I_{j}(f)=I_{j}, 0<k<2 N$ by

$$
\begin{cases}I_{j}=\left\{t_{j}\right\} & \text { if } j \text { is even, } \\ I_{j}=\left(t_{j-1}, t_{j+1}\right) & \text { if } j \text { is odd. }\end{cases}
$$

We thus use even numbers to index the relative extrema and odd numbers to index the maximal monotone intervals. Note that the interval is the disjoint union of the $I_{j}$ 's. This indexing system greatly simplifies the combinatorics on which the classification system is based.

For each $x$ in $I$, the itinerary of $x$ with respect to $f$ is the infinite sequence $\mathbf{a}_{f}(x)=\left(a_{0}(x), a_{1}(x), \ldots, a_{n}(x), \ldots\right)$ defined by $a_{n}(x)=j$ iff $f^{n}(x)$ is in $I_{j}$, where $f^{n}$ is $f$ composed with itself $n$ times. Since $\left\{I_{k}: 0 \leq k \leq 2 N\right\}$ is a partition of $I, a_{n}(x)$ is uniquely defined. For even $k$ we define $\mathbf{e}(k)=\mathbf{a}_{f}\left(t_{k}\right)$, and $e_{j}(k)=a_{j}\left(t_{k}\right)$, and we let $E_{f}=\{\mathbf{e}(k): k$ even $\}$.

3. Definition. Let $N$ be a positive integer and let sgn be a function with domain $\{-1,0,1,2, \ldots, 2 N, 2 N+1\}$ satisfying $\operatorname{sgn}(j)=0$ if $j$ is even and $\operatorname{sgn}(j)=(-1)^{b+(j+1) / 2}$ if $j$ is odd, where $b=0$ or 1 is fixed. In other words, sgn is one of two possible functions which is zero on the even integers and alternates between 1 and -1 on the odd integers. This is a convenient way of coding 
whether a function is increasing or decreasing on an interval $I_{j}, j$ odd. Putting -1 and $2 N+1$ in the domain will make the statements of some theorems cleaner. Let $S_{N}$ be the set of all infinite sequences $\mathbf{a}=\left(a_{0}, a_{1}, \ldots, a_{k}, \ldots\right)$ such that $\operatorname{range}(\mathbf{a})$ is contained in $\{0,1,2, \ldots, 2 N\}$. If $\mathbf{a} \neq \mathbf{b}$ both in $S_{N}$, then define $\mathbf{a}<\mathbf{b}$ if

$$
\left(\prod_{i=0}^{k-1} \operatorname{sgn}\left(a_{i}\right)\right) a_{k}<\left(\prod_{i=0}^{k-1} \operatorname{sgn}\left(b_{i}\right)\right) b_{k}
$$

where $k$ is least such that $a_{k} \neq b_{k}$. < is easily seen to be a strict partial ordering. Define $\leq$ is the obvious way, i.e. $\mathbf{a} \leq \mathbf{b}$ iff either $\mathbf{a}=\mathbf{b}$ or $\mathbf{a}<\mathbf{b}$. Note that the ordering is not linear, and $\mathbf{a}$ and $\mathbf{b}$ are related with respect to $<$ iff no even numbers appear in $\mathbf{a}$ and $\mathbf{b}$ prior to the first point of disagreement.

If $\mathbf{a}$ is a finite sequence and $\mathbf{b}$ is a finite or infinite sequence, then $\mathbf{a b}$ indicates the concatenation of the sequences $\mathbf{a}$ and $\mathbf{b}$, and $\mathbf{a}^{n}$ means aaa $\cdots \mathbf{a}$ ( $n$ times). $\mathbf{a}^{*}$ will be a repeated infinitely many times. Given a sequence $\mathbf{a}=\left(a_{0}, a_{1}, a_{2}, \ldots\right)$, the shift operation $S$ is defined by $S(\mathbf{a})=\left(a_{1}, a_{2}, \ldots\right)$. $S^{n}$ is $S$ repeated $n$ times. We say $\mathbf{b}$ is a shift of $\mathbf{a}$ if $\mathbf{b}=S^{n}(\mathbf{a})$ for some $n$, and $\mathbf{b}$ is a preshift of $\mathbf{a}$ if $\mathbf{a}=S^{n}(\mathbf{b})$ for some $n$ (equivalently, $\mathbf{b}=\mathbf{c a}$ for some c). An element a of $S_{N}$ is called totally odd iff $a_{i}$ is odd for all $i$. If $\mathbf{a}$ is a sequence of length greater than $n$, then $a \nmid n$ will abbreviate $\mathbf{a} \mid\{0,1,2, \ldots, n-1\}$.

Clearly, there are some elements of $S_{N}$ which cannot be the itinerary of any point $x$ for any pwm $f$. A trivial example is $00\left(1^{*}\right)$. The following theorem gives some easy restrictions.

4. Theorem. Let $f$ be pwm, $N=N_{f}$, sgn= the sign function for $f, E=$ $E_{f}=(\mathbf{e}(0), \mathbf{e}(2), \ldots, \mathbf{e}(2 N))$. Then for any $x$ in $I$ with $\mathbf{a}=\mathbf{a}_{f}(x)$ :

(a) $\mathbf{a}_{f}(f(x))=S(\mathbf{a})$.

(b) If $x<y$ then $\mathbf{a}_{f}(x) \leq \mathbf{a}_{f}(y)$.

(c) If $a_{k}$ is even, then $S^{k}(\mathbf{a})=\mathbf{e}(k)$.

(d) If $i=\mathbf{a}_{k}$ is odd, then

$$
\begin{gathered}
(\mathbf{e}(i-1)) \leq S^{k+1}(\mathbf{a}) \leq S(\mathbf{e}(i+1)) \quad \text { if } \operatorname{sgn}(i)=1, \\
S(\mathbf{e}(i-1)) \geq S^{k+1}(\mathbf{a}) \geq S(\mathbf{e}(i+1)) \quad \text { if } \operatorname{sgn}(i)=-1 .
\end{gathered}
$$

(e) If $k$ is even and $\left|e_{i}(k)-a_{i}\right| \leq 1$ for all $i, a_{0}$ is odd, and $e_{j}(k)$ is even, than $a_{j}$ is odd.

Proof. (a) and (c) are obvious. For (b) assume $\mathbf{a}_{f}(x)>\mathbf{a}_{f}(y)$ and let $k$ be least such that $a_{k}(x)=a_{k}(y)$. Then $a_{k}(x)>a_{k}(y)$ iff the images of $x$ and $y$ switch an even number of times when $f$ is applied $k$ times. (e) is an easy exercise. For (d) note that if $i=a_{0}$ is odd, then by ( $\mathrm{b}$ )

$$
\begin{gathered}
S(\mathbf{e}(i-1)) \leq S(\mathbf{a}) \leq S(\mathbf{e}(i+1)) \quad \text { if } \operatorname{sgn}(i)=1, \\
S(\mathbf{e}(i-1)) \geq S(\mathbf{a}) \geq S(\mathbf{e}(i+1)) \quad \text { if } \operatorname{sgn}(i)=-1 .
\end{gathered}
$$


Applying this $k$ times gives (d), by an easy application of (a).

5. Definition. Let $f$ be pwm. For each sequence a of length $n$, let $I_{a}(f)=$ $I_{a}=\left\{x:\left(\mathbf{a}_{f}(x)\right) \mid n=\mathbf{a}\right\}$. Note that if $\mathbf{a}$ is a sequence of length 1 with $a_{0}=j$, then $I_{a}=I_{j}$ as defined before. If $\mathbf{a}$ is infinite, let $I_{a}=\left\{x: \mathbf{a}_{f}(x)=\mathbf{a}\right\}$, and note that $I_{a}$ is the intersection of the $I_{a \mid n}$ 's.

6. Proposition. If $\mathbf{a}$ is an initial segment of $\mathbf{b}$, then $I_{b}$ is a subset of $I_{a}$.

7. Proposition. If a is finite and totally odd, then $I_{a}$ is an open interval (possibly empty). If a is not totally odd, then $I_{a}$ is either a singleton or empty.

8. Lemma. Let $f$ and $g$ be pwm such that $E_{f}=E_{g}$ and $f$ and $g$ have the same sgn function. Then for every finite sequence a from $S_{N}, I_{a}(f)$ is nonempty iff $I_{a}(g)$ is nonempty.

Proof. By induction on the length of a. It is clearly true if a has length 1. Suppose the lemma is true for all sequences of length $k$, and let a be a sequence of length $k+1$. Let $\mathbf{a}=(i) \mathbf{b}$, where $\mathbf{b}$ is a sequence of length $k$ and $0 \leq i \leq$ $2 N$. If $i$ is even, then the result is easy, for $I_{a}(f)$ and $I_{a}(g)$ are nonempty just in case $\mathbf{a}$ is the restriction of $\mathbf{a}_{f}\left(t_{i}(f)\right)=\mathbf{a}_{g}\left(t_{i}(g)\right)$ to $k+1$. Thus, assume $i$ is odd, $I_{a}(f)$ is nonempty, and let $x$ be such that $\mathbf{a}_{f}(x) \mid k+1=\mathbf{a}$. Then $t_{i-1}(f)<x<t_{i+1}(f)$ and $\mathbf{e}_{i-1}<\mathbf{a}<\mathbf{e}_{i+1}$. If $\operatorname{sgn}(i)=-1$, then $S\left(\mathbf{e}_{i-1}\right) \geq$ $S(\mathbf{a})=b \geq S\left(\mathbf{e}_{i+1}\right)$. Since $I_{b}(f)$ is nonempty (it contains $\left.f(x)\right), I_{b}(g)$ is also nonempty by the induction hypothesis. Since $S\left(\mathbf{e}_{i-1}\right) \geq S(\mathbf{a})=b \geq S\left(\mathbf{e}_{i+1}\right)$, it is easy to see that $I_{b}(g)$ must contain at least one point, say $y$, such that $g\left(t_{i-1}(g)\right)>y>g\left(t_{i+1}(g)\right)$. By the intermediate value theorem there must be a $z$ such that $t_{i-1}(g)>z>t_{i+1}(g)$ and it is clear that $\mathbf{a}_{g}(z) \mid(k+1)=\mathbf{a}$. The remaining cases are all symmetric to the one just described.

The word "finite" in the statement of Lemma 8 is necessary. See Figure 2 for an example.

9. Definition. A pwm function $f$ on $I$ is expanding iff whenever $x<y$ and $f$ is monotone on $[x, y]$ then

$$
\frac{|f(y)-f(x)|}{|y-x|}>1
$$

(Expanding is sometimes defined with the difference quotient being bounded away from 1 , but this slightly weaker definition will be more than we need.) We will say that $f$ is weakly expanding iff $\mathbf{a}_{f}$ is one-to-one, i.e. different points have different itineraries.

10. Proposition. Expanding implies weakly expanding.

Proof. Let $U$ be any open interval. Then it is routine to show that $f^{j}(U)$ intersects $I_{i}=\left\{t_{i}\right\}$ for some $j$ and even $i$. The rest is easy.

11. Theorem. If $f$ and $g$ are weakly expanding, then $f \sim g$ iff $E_{f}=E_{g}$.

Proof. For each positive integer $n$, let $h_{n}$ be an order-preserving homeomorphism of $I$ such that for each $a$ of length $n, h_{n}$ maps $I_{a}(f)$ linearly onto 
$I_{a}(g)$. This can be done by Proposition 7 and Lemma 8. Since $f$ and $g$ are weakly expanding, the lengths of the intervals $I_{a}(f)$ and $I_{a}(g)$ approach 0 as $n$ gets large. Thus the sequence $\left(h_{n}\right)$ converges uniformly to an order-preserving homeomorphism $h$, and it is easy to check that $f=h^{-1} g h$.

The above results show how complete our information is for the case of expanding pwm maps. Their strong conjugacy class is completely specified by the itineraries of the turning points, and the function witnessing strong conjugacy is just the function which takes each point to the (unique) point having the same itinerary for the other function.

The main difficulty in getting a complete set of invariants for all pwm functions is that nonexpanding functions can behave in a more pathological way than expanding ones, at least in certain respects. Since the nonexpanding functions include all functions having a finite attracting orbit there are many functions with dynamically interesting properties which are not included among the expanding maps. Thus, if a classification is to be found which will work for these functions, additional invariants need to be identified.

\section{MONOTONE FUNCTIONS ON THE INTERVAL}

Much of the additional behavior of pwm functions (i.e. that behavior which cannot be described by the itineraries alone) is already present in the case of monotone functions (note that a monotone function cannot be even weakly expanding). Thus, we will spend this section going over the monotone case, as that will be one of the components of the eventual classification. It is easy to see that there are cardinality of the continuum many pairwise nonconjugate orderpreserving homeomorphisms on $I$. For each subset $X$ of the set of natural numbers $\{0,1,2,3, \ldots\}$ define a function $f=f_{x}$ such that the fixed points of $f$ are exactly 0 and $\{1 /(n+1): n=0,1,2,3, \ldots\}$ and let $f(x)>x$ on $(1 /(n+2), 1 /(n+1))$ if $n \in X$ and $f(x)<x$ on $(1 /(n+2), 1 /(n+1))$ if $n \notin X$. Then it is easily seen that if $X \neq Y$ then $f_{X}$ is not conjugate with $f_{Y}$. On the other hand, the above trick (and minor variations) is about all that is available for constructing nonconjugate order homeomorphisms, as we shall see below. The following theorem is presumably a part of folklore, although I do not know a specific reference. The proof has been included as a simple example of the "back-and-forth method" that will be useful below in more complicated situations.

12. Theorem. Let $f, g$ be homeomorphisms of $I$ such that $f(0)=g(0)=0$, $f(1)=g(1)=1$, and $f(x)>x, g(x)>x$ for all $x$ such that $0<x<1$. Then $f$ and $g$ are strongly conjugate.

Proof. Let $\left\{U_{n}: n=1,2,3, \ldots\right\}$ enumerate a countable basis of $I$. We define functions $h_{n}$ and subsets $X_{n}, Y_{n}$ of $I$ by induction on $n$ satisfying the following hypotheses:

(a) If $m<n$ then $X_{m} \subseteq X_{n}$ and $Y_{m} \subseteq Y_{n}$.

(b) $X_{n}$ is closed under $f$ and $f^{-1}$, and $Y_{n}$ is closed under $g$ and $g^{-1}$. 
(c) $X_{n}$ and $Y_{n}$ are countable and if $0<u<v<1$, then $(u, v)$ contains only finitely many points $X_{n}$ and $Y_{n}$.

(d) $h_{u}$ is an order-preserving bijection from $X_{n}$ to $Y_{n}$ which extends $h_{m}$ for $m<n$.

(e) If $n \geq 2 k$ and $K>0$ then $X_{n}$ and $Y_{n}$ both intersect $U_{k}$.

(f) $f(x)=h_{n}^{-1} g h_{n}(x)$ for every $x$ in $X_{n}$.

We start by letting $X_{0}=Y_{0}=\{0,1\}, h_{0}(0)=0, h_{0}(1)=1$. Now suppose $n>0$ and $X_{n}, Y_{n}, h_{n}$ have been defined for $m<n$ so that the induction hypotheses hold.

Case 1. $n$ odd, say $n=2 k+1$. Pick any element of $U_{k}-X_{n-1}$, say $x$, and let $X_{n+1}=X_{n},\left\{f^{j}(x): j=\ldots,-3,-2,-1,0,1,2,3, \ldots\right\}$. Let $u$ be greatest and $v$ least such that $u<x<v$ and $u, v$ both in $X_{n}$. Pick any point in $(u, v)$, say $y$, and let $Y_{n+1}=Y_{n},\left\{g^{j}(y): j=\ldots,-3,-2,-1,0,1,2\right.$, $3, \ldots\}$. Finally, extend $h_{n}$ to $h_{n+1}$ by defining $h_{n+1}\left(f^{j}(x)\right)=g^{j}(y)$.

Case 2. $n$ even, say $n=2 k$. Same as Case 1, except that $Y_{n+1}$ is defined first and then $h_{n}^{-1}$ (instead of $h_{n}$ ) is then used to define $X_{n}$. The resulting $h_{n}$ 's combine to give a function $h$ which is order-preserving from a dense subset of $I$ to another dense subset of $I$, which easily induces the desired homeomorphism.

Note that this theorem works for intervals other than $[0,1]$, including open or half-open intervals (assuming, of course, that the open and closed ends match). As an immediate corollary, we get a criterion which tells us whether or not two orientation-preserving homeomorphisms of the interval are strongly conjugate.

13. Corollary. Let $f$ and $g$ be orientation-preserving homeomorphisms of $I$. Let $L(f)$ be the set of all subintervals $J$ of $I$ (open, closed, or half-open) which are maximal with respect to the property:

Exactly one of the following occurs on $J$ :

1. $f(x)=x$ for all $x$ in $J$,

2. $f(x)<x$ for all $x$ in $J$, or

3. $f(x)>x$ for all $x$ in $J$.

For each $J$ in $L(f)$, define $r(J)$ to be $0,-1$, or 1 , depending on whether (respectively) 1, 2, or 3 above holds. Define $L(g)$ and $r^{\prime}$ on $L(g)$ in the same way. Then $f$ and $g$ are strongly conjugate if and only if there is a bijection $h^{\prime}: L(f) \rightarrow L(g)$ such that for every $J<K$ both in $L(F), h^{\prime}(J)<h^{\prime}(K)$ (where $J<K$ means that every element of $J$ is less than every element of $K$ ), and for every $J$ in $L(f), r(J)=r^{\prime}\left(h^{\prime}(J)\right)$.

Proof. If $f$ and $g$ are strongly conjugate, and $h$ witnesses this, then $h^{\prime}(J)=$ $h[J]$ clearly works. In the other direction, if $h^{\prime}$ is as above, define an orientation-preserving homeomorphism $h$ such that $h[J]=h^{\prime}(J)$ for all $J$ in $L(f)$. Define $h: J \rightarrow h^{\prime}(J)$ to be linear map if $r(J)=0$, and if $r(J)$ is -1 , or 1 , use a trivial modification of Theorem 12 to define $h: J \rightarrow h^{\prime}(J)$ such that 
$h^{-1} f h(x)=g(x)$ for all $x$ in $J$. Then $h$ is easily seen to be an orientationpreserving homeomorphism such that $g=h^{-1} f h$.

Suppose we now consider two orientation-reversing homeomorphisms $f$ and $g$ of $I$. Then it is easy to check that $f \sim g$ iff $f^{2}\left|[0, x] \sim g^{2}\right|[0, y]$, where $x$ and $y$ are the fixed points of $f$ and $g$ respectively (an easy proof which is left to the reader). Thus Corollary 13 above could be modified in a routine way to get a criterion which decides if any two homeomorphisms of $I$ are strongly conjugate.

The above observation suggests an additional invariant. Note that if $f$ is a homeomorphism, then every point in the interior of $I$ has the same itinerary. The above observations about homeomorphisms could then be reworded (very vaguely) as: "Intervals in which all points have the same itinerary should map to each other in the same way" (something that is obvious from the homeomorphism case alone). However, there is one additional piece of information which needs to be taken care of in addition.

Suppose $f$ and $g$ are pwm functions such that $E_{f}=E_{g}$ and we want to know whether or not $f$ and $g$ are strongly conjugate. Suppose $f$ and $g$ both have points having itinerary $\mathbf{b}=\mathbf{a}^{*}$ for some finite $\mathbf{a}$ of length $n$. Then clearly $f^{n} \mid I_{a}(f)$ must be conjugate to $g^{n} \mid I_{a}(g)$. This condition, though necessary, is definitely not sufficient to get $f$ and $g$ strongly conjugate, however, as the following two examples in the illustration below shows.

In both examples we have $E_{f}=E_{g}=\left\{0\left(9^{*}\right), 2\left(0^{*}\right), 4\left(9^{*}\right), 6^{*}, 8^{*}\right\}$ and if $\mathbf{a}$ is any finite sequence of length $n$ then it is easy to see that $f^{n} \mid I_{a^{*}}(f)$ is conjugate to $g^{n} \mid I_{a^{*}}(g)$. However, it is clear by inspection that the two functions are not conjugate, because the first two relative maxima do not map in the same way. Thus we are not only going to have to make sure that intervals having the same itinerary map in the "same" way, but whenever some of the turning points map (or eventually map under iterations) into such an interval, we have

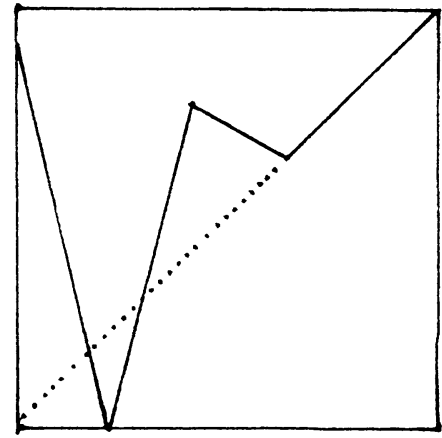

$f$

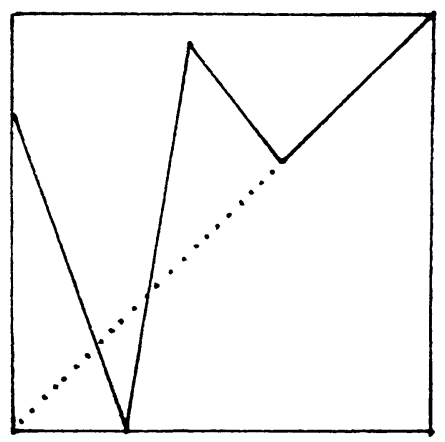

$g$ 
to have a way of keeping track of where the images of these extrema land. The next definition gives us a way of doing that.

14. Definition. If $f$ and $g$ are continuous functions on the intervals $J$ and $K$ respectively, and $F$ and $G$ are finite subsets of $J$ and $K$ respectively, then we will say that $(f, F)$ and $(g, G)$ are strongly conjugate iff there is an order-preserving homeomorphism $h: J \rightarrow K$ such that $f=h^{-1} g h$ and $h(F)=G$.

It is clear that this is a much stronger property than $f$ and $g$ just being strongly conjugate, because the sets $F$ and $G$ are already defining part of the homeomorphism $h$. It turns out that a classification of the strong conjugacy classes of $(f, F)$ for (strictly) monotone (but not necessarily onto) maps $f$ is exactly what we need to complete the classification of the pwm maps. We will have a way of combining this with what has already been done with itineraries to complete the result, and it will really not make any difference what classification scheme is used for the pairs $(f, F), f$ monotone, $F$ finite. Rather than going into details at this point, we will assume for the moment that we have a classification scheme for all such pairs $(f, F)$, so that we can proceed fairly quickly to the main result. The next few sections are completely independent of whatever classification scheme is used for the $(f, F)$-equivalence classes. Later, after we have proven the main result, we will show how to get a classification scheme for the $(f, F)$-classes which avoids most, if not all, of the equivalenceclass copout.

From this point on we assume we have some classification of the strong conjugacy classes of all pairs $(f, F)$, where $f$ is a (strictly) monotone function on $J$ for some interval $J$ and $F$ is a finite subset of $J$. It does not really matter which of the many possible classification schemes for $(f, F)$ is used here, but it will be easier to describe the main classification if we assume that we are taking one representative $(f, F)$ from each conjugacy class and using that as our classification of the monotone maps. We assume that our classification of all such $(f, F)$ includes all strictly monotone functions on intervals which may be open at one or both ends, and that the empty interval is included as one possibility.

15. Definition. Fix a classification of all pairs $(f, F)$ such that $f$ is a monotone continuous function on some interval (which could be closed, open, halfopen-half-closed, and could also be a singleton or empty) and $F$ is a finite subset (possibly empty) of the domain of $f$. Let $P=\{Q(f, F): f$ is a monotone function on some interval $J$ and $F$ is a finite subset of $J\}$, where $Q(f, F)$ is any invariant which classifies $(f, F)$ according to its strong conjugacy class. For convenience, the element of $P$ which represents $(f, F)$ where $f$ is a function from a point $\{x\}$ to itself will be called $Q_{1}$ if $F$ is the empty set and $Q_{1}^{*}$ if $F=\{x\}$. The element which represents the empty function will be called $Q_{0}$. 
16. Definition. Two elements $\mathbf{a}$ and $\mathbf{b}$ of $S_{N}$ will be called equivalent, abbreviated $\mathbf{a} \# \mathbf{b}$, iff there are integers $m$ and $n$ such that $S^{m}(\mathbf{a})=S^{n}(\mathbf{b})$, i.e., some final segment of $\mathbf{a}$ is the same as some final segment of $\mathbf{b}$. Given any $\mathbf{a}$, the equivalence class of a modulo \# will be denoted [a]. In most cases we will only be interested in a subset of [a] consisting of sequences $\mathbf{b}$ which are relevant to the current problem. For example, if $f$ is pwm, we let [a] $]_{f}$ consist of all elements b of [a] such that $I_{a \mid n}(f)$ is nonempty for every positive integer $n$, and write [a] instead of [a] $]_{f}$ if there is no danger of confusion. These equivalence classes are just the minimal sets which are closed under shifts and preshifts. Let $k$ be even, $k \leq N$. Given $f$ and $E=E_{f}$, we define $G_{k}=G_{k}(f)=\{\mathbf{e}(i): i$ even, $\mathbf{e}(i) \# \mathbf{e}(k)\}$, and define $G[\mathbf{a}]=G_{k}$ if $\mathbf{a} \# \mathbf{e}(k)$ (with $G[\mathbf{a}]$ left undefined if there is not such $k) . G_{k}$ is called periodic if some element of $[\mathbf{e}(k)]$ (i.e. some final segment of $\mathbf{e}(k))$ is periodic, and is called aperiodic otherwise.

\section{THE CLASSIFICATION}

We are now in a position to define the promised complete set of invariants for the strong conjugacy classes of the pwm functions.

17. Definition. If $f$ is a pwm function, $N=N_{f}, E=E_{f}, \operatorname{sgn}=\operatorname{sgn}_{f}$ is the sign function for $f, H_{f}=\left\{[\mathbf{a}]_{f}\right.$ : for all $n, I_{a \mid n}(f)$ is nonempty $\}$, we define the function $q=q_{f}$ having domain $H=H_{f}$ as follows (where, as mentioned above, we write [a] for $\left.[\mathbf{a}]_{f}\right)$ :

Case 1. [a] has not totally odd element. Let $q[\mathbf{a}]=\left(Q_{1}^{*}, r\right)$ where $r$ is the constant map from $G[\mathbf{a}]$ to the point of $Q_{1}^{*}$.

Case 2. [a] has a totally odd periodic element. Pick d finite of smallest possible length so that $\left[\mathbf{d}^{*}\right]=[\mathbf{a}]$, and such that $\mathbf{d}^{*}$ is least possible in the $<$ ordering of $S_{N}$. Let $J=\left\{x: \mathbf{a}_{f}(x)=\mathbf{d}^{*}\right\}$ and let $n$ be the length of $\mathbf{d}$. Then $f^{n}$ maps $J$ into itself. For each $k$ such that $[\mathbf{c}(k)]=[\mathbf{a}]$, we let $j(k)$ be least such that $S^{j(k)}(\mathbf{c}(k))=\mathbf{d}^{*}$ and let $F=\left\{f^{j(k)}\left(t_{k}\right):[\mathbf{c}(k)]=[\mathbf{a}]\right\}$. We then define $q[\mathbf{a}]=\left(Q\left(f^{n} \mid J, F\right), r\right)$, where $r(c(k))=f^{j(k)}\left(t_{k}\right)$ for $c(k)$ in $G[\mathbf{a}]$.

Case 3. [a] has a totally odd aperiodic element. If every element of [a] is totally odd then let $q[\mathbf{a}]=(Q(g, G), r)$ where $G$ and $r$ are empty and $g$ is a constant function whose domain is either a point or a nontrivial closed interval, depending on whether $I_{a}$ is a point or a nontrivial closed interval. If [a] has an element which is not totally odd, i.e. $G[\mathbf{a}]$ is nonempty, then pick d maximal so that $[\mathbf{d}]=[\mathbf{a}]$, $\mathbf{d}$ is totally odd, and $\mathbf{d}$ is a shift of every element of [a] which is not totally odd. Let $J=\left\{x: \mathbf{a}_{f}(x)=\mathbf{d}\right\}$. For each $k$ such that $[c(k)]=$ [a], we let $j(k)$ be least such that $S^{j(k)}(c(k))=\mathbf{d}$ and let $F=\left\{f^{j(k)}\left(t_{k}\right):[c(k)]=[\mathbf{a}]\right\}$. We then define $q[\mathbf{a}]=((\mathrm{id}, F), r)$, where id is the identity function and $r(c(k))=f^{j(k)}\left(t_{k}\right)$ for $c(k)$ in $G[\mathbf{a}]$. 
This completes the definition of $q_{f}$. Note that for each [a] in $H, q_{f}[\mathbf{a}]=$ $(Q, r)$ for some $Q=Q(g, G)$ for some $g, G$ and some $r: G[\mathbf{a}] \rightarrow G$. Then the classification of $f$, denoted $C_{f}$, is the triple $\left(\operatorname{sgn}_{f}, E_{f}, q_{f}\right)$.

18. Main Theorem. $C_{f}$ is a complete set of invariants for the strong conjugacy classes of piecewise monotone functions, i.e. $f \sim g$ if and only if $C_{f}=C_{g}$.

Proof. We define an order-preserving homeomorphism $h$ of $I$ such that $f=$ $h^{-1} g h$. Let $C=C_{f}=C_{g}, E=E_{f}=E_{g}, \operatorname{sgn}=\operatorname{sgn}_{f}=\operatorname{sgn}_{g}, q=q_{f}=q_{g}$. Note that since $H_{f}=H_{g}, I_{a}(f)$ is nonempty iff $I_{a}(g)$ is nonempty for every a (finite or infinite), so $[\mathbf{a}]_{f}=[\mathbf{a}]_{g}$, and we can write [a] without fear of ambiguity. We begin by requiring that $h\left(I_{a}(f)\right)=I_{a}(g)$ for every a in $E$, and we now have to specify how $h$ maps $I_{a}(f)$ to $I_{a}(g)$ in those cases where $I_{a}(f)$ and $I_{a}(g)$ are not singletons.

Case 1. [a] is periodic. Let $\mathbf{d}$ be <-least such that $\left[\mathbf{d}^{*}\right]=[\mathbf{a}], \mathbf{d}^{*}$ is periodic, and $\mathbf{d}$ is not repeating. Let $k$ be the iength of $\mathbf{d}$. If $\mathbf{d}$ is not totally odd, then $I_{a}(f)$ and $I_{a}(g)$ are singletons and the definition of $h \mid I_{a}(f)$ is obvious, so assume $\mathbf{d}$ is totally odd. Let $B(\mathbf{a}, f)=\{t: t$ is an extremum of $f$ and $a_{f}\left(f^{i}(t)\right)=\mathbf{d}^{*}$ for some $\left.i\right\}$, and define $B(\mathbf{a}, g)$ similarly. For each $t$ in $B(\mathbf{a}, f)$, let $j(f, t)$ be the least $j$ so that $\mathbf{a}_{f}\left(f^{j(f, t)}(t)\right)=\mathbf{d}^{*}$ and define $j(g, t)$ similarly. Now let $S(f)$ be the set of all $f^{j(f, t)}(t)$ for $t$ in $B(\mathbf{a}, f)$ and similarly for $S(g)$. Thus, since $q_{f}[\mathbf{a}]=q_{g}[\mathbf{a}]$, we can define $h$ with domain $I_{d^{*}}(f)$ and range $I_{d^{*}}(g)$ such that $f k=h^{-1} g k h$ on $I_{d^{*}}(f)$ such that $h(S(f))=h(S(g))$ and this is easily used to define $h$ on $I_{b}(f)$ for all $\mathbf{b}$ such that $\mathbf{b}$ is a shift of $\mathbf{d}^{*}$, i.e. for each integer $i$ and each $x$ in $I_{d^{*}}(f)$ let $h\left(f^{i}(x)\right)=g^{i}(h(x))$. This defines $h$ on $I_{b}(f)$ for each shift $\mathbf{b}$ of $\mathbf{d}^{*}$. Doing the same for preimages works because $h$ maps $S(f)$ to $S(g)$.

Case 2. [a] is aperiodic. Let $\mathbf{d}$ be maximal so that [a] $=[\mathbf{d}]$, $\mathbf{d}$ totally odd, and every element of [a] which is not totally odd is a preshift of $\mathbf{d}$. Since $\mathbf{d}$ is aperiodic such a d must exist. We define $S(f)$ and $S(g)$ as before and define $h$ from $I_{f}(\mathbf{d})$ to $I_{g}(\mathbf{d})$ so that $h(S(f))=S(g)$, and then work as in Case 1 . This completes the definition $h$. Since $h$ was constructed so that $f=h^{-1} g h$ on $\left\{x: \mathbf{a}_{f}(x)=\mathbf{b}\right.$ for some $\mathbf{b}$ such that $\left.[\mathbf{a}]=[\mathbf{b}]\right\}$, it is clear that $f=h^{-1} g h$. Furthermore, since $h$ is an order preserving homeomorphism from $I_{a}(f)$ to $I_{a}(g)$ for each $\mathbf{a}$ in $E$ and $E_{f}=E_{g}$, it is clear that $h$ is a homeomorphism of $I$.

This completes the main theorem, subject to a small amount of ambiguity regarding the classification of the pairs $(f, F)$, where $f$ is (strictly) monotone and $F$ is a finite subset of the domain of $f$. We now give more details about how these can be related to countable linear orderings.

19. Definition. A triple $(L,<, r)$ will be called a labelled-ordering, abbreviated $L$-ordering, iff $(L,<)$ is a countable linear ordering and $r$ is a function with domain $L$ and range $\{-1,0,1,2\}$ having the following properties: 
(a) If $x$ and $y$ are adjacent in $L$, then $r(x)=r(y)$.

(b) If $r(x)=2$, then $x$ is an endpoint of $L$.

We emphasize here that countable does not necessarily mean infinite, so finite (and empty) $L$-orderings are allowed. If $L=(L,<, r)$ and $L^{\prime}=\left(L^{\prime},<^{\prime}, r^{\prime}\right)$ are $L$-orderings, we say that $L$ and $L^{\prime}$ are isomorphic iff there is an orderpreserving bijection $h$ from $L$ onto $L^{\prime}$ such that $h(r(x))=r^{\prime}(h(x))$ for all $x$ in $L$.

20. Definition. Let $f$ be an increasing function on $I$. We define a $L$-ordering $L_{f}=(L,<, r)$ such that $L$ is the set of all nontrivial (i.e. more than one point) subintervals of $I$ which are maximal with respect to either the property of being the identity map on the interval or the property of having no fixed points on the interval. These intervals are ordered in the obvious way. If $f(0)=0$ and $J$ is the least element of $L$, then $r(J)=2$, and similarly, if $f(1)=1$ and $J$ is the largest element of $L$, then $r(J)=2$. For all other members $J$ of $L$ we define

$$
r(x)= \begin{cases}1 & \text { if } f(x)>x \text { on } J \\ 0 & \text { if } f(x)=x \text { on } J \\ -1 & \text { if } f(x)<x \text { on } J\end{cases}
$$

21. Theorem. If $f$ and $g$ are increasing functions on $I$, then $f$ and $g$ are strongly conjugate iff $L_{f}$ is isomorphic to $L_{g}$.

Proof. Let $h$ be the isomorphism of $L_{f}$ and $L_{g}$. Define an order-preserving homeomorphism $h^{\prime}$ on $I$ by first using $h$ to decide which intervals map to which intervals. On intervals $J$ in $L_{f}$ such that $r(J)=0$, let $h^{\prime} \mid J$ be any order-preserving homeomorphism from $J$ to $h(J)$. On intervals $J$ in $L_{f}$ such that $r(J)$ is -1 or 1 , use a trivial modification of Theorem 12 to define $h^{\prime}$ on $J$. If $r(J)=2$, then the same back-and-forth argument as in Theorem 12 gets a conjugacy between $J$ and $h(J)$. The function $h^{\prime}$ is now defined on a dense subset of $I$, and is trivially extended to the desired conjugacy.

Note that given any nonempty $L$-ordering $L$, it is easy to construct an increasing function $f$ on $I$ such that $L_{f}=L$ (just replace each element of $L$ by a nonempty open interval, add points as in Dedekind cuts to make the resulting ordering homeomorphic to $[0,1]$, and define the function according to $r$ ), so there is a one-to-one correspondence between the strong conjugacy classes of increasing functions on $I$ and the isomorphism classes of nonempty $L$-orderings. This gives us a strong indication that what I have called the "equivalence class copout" cannot be entirely avoided, for set-theoretic difficulties would make even a classification of the isomorphism classes of countable linear orderings (without a labelling) extremely difficult (or perhaps impossible) without using equivalence classes of some other set-theoretic trick.

If we want to classify the pairs $(f, F)$, then more information is needed. For example, suppose $f$ is a homeomorphism of $I$ such that $f(0)=0, f(1)=1$, and $f(x)>x$ for $0<x<1$. Then if $s$ and $t$ are points in $I$, then $(f,\{s\})$ 
and $(f,\{t\})$ may not be conjugate, for one of $s$ and $t$ might be an endpoint and the other a nonendpoint. The situation gets even more complicated with two or more points. To determine the strong conjugacy class of $(f,\{s, t\})$, one needs to know the answer to such questions as "Is $f^{n}(s)=t$ for some $n$ ? and "If $s<t$, what is the smallest $n$ such that $f^{n}(s)>t$ ?". However, this additional information can be described in a uniform way that adds only a finite amount of additional information to the labelled ordering.

22. Definition. An intertwining is a pair $(n, V)$ such that $n$ is a positive integer and $V$ is a set of nonnegative integers such that every congruence class modulo $n$ has at least one representative in $V$ and 0 is in $V$. If $J$ is an open interval of reals and $f$ is an order-preserving homeomorphism of $J$ such that $f(x)>x$ for all $x$ in $J$ and $F$ is a finite subset of $J$, then $\mathbf{V}(f, F)$ is defined to be any intertwining $(n, V)$ such that for some order-preserving homeomorphism from $J$ onto $\mathbf{R}, h f h^{-1}(x)=x+n$ for all $x$ in $J$, and $h(F)=V$.

\section{Proposition. Such a $\mathbf{V}(f, F)$ exists and is uniquely defined.}

Proof. Let $x_{0}$ be the smallest element of $F$, let $X$ be the smallest subset of $J$ which is closed under $f$ and $f^{-1}$ and let $n$ be the number of disjoint orbits of $X$. Then $X$ is a discrete subset of $J$, so it is easy to use the back-and-forth argument outlined above to get a conjugacy $h$ between $f$ and the function $g(x)=x+n$ on $\mathbf{R}$ taking $x_{0}$ to 0 and $X$ to the set of integers. Then let $V=h(F)$ and it is easy to see that $(n, V)$ is an intertwining satisfying the desired properties. That no other intertwining has these properties is an easy exercise.

24. Theorem. Let $J$ be an open interval and let $f$ and $g$ be two homeomorphisms of $J$ such that $f(x)>x$ and $g(x)>x$ for all $x$. Let $F$ and $G$ be two finite subsets of $J$. Then $(f, F)$ and $(g, G)$ are strongly conjugate iff $\mathbf{V}_{f}=\mathbf{V}_{g}$.

Proof. An easy exercise.

Combining the above ideas of $L$-orderings and intertwinings to get a classification scheme for the strong conjugacy classes $(f, F)$ for arbitrary monotone $f$ is now routine. In addition to assigning an $L$-ordering to $f$, for each maximal interval $J$ for which the graph of $f$ lies above the diagonal we have to assign the intertwining $\mathbf{V}(f, F \cap J)$, and if $J$ is maximal such that the graph lies below the diagonal we use $\mathbf{V}(-f(-x), F \cap J)$. A finite number of additional pieces of information are also needed to describe the elements of $F$ which are fixed by $f$. Putting this all together gives a classification of all strong conjugacy classes $(f, F)$ for all orientation-preserving homeomorphisms $f$ of $I$ and all finite subsets $F$ of $I$. Extending this to the cases where $f$ is either decreasing or not onto is an easy extension of the above. If $f$ is decreasing, then we can use the above observation that two decreasing functions $f$ and $g$ on $[0,1]$ 
are strongly conjugate iff $f^{2} \mid[0, x]$ and $g^{2} \mid[0, y]$ are strongly conjugate, where $x$ and $y$ are the fixed points of $f$ and $g$ respectively. Of course, additional labels are required to indicate whether a member of $F$ came from $[0, x]$ or $(x, 1]$, but this annoying detail is routine and is left to the reader. If $f$ is not onto, then a label at each end of the interval will take care of this. The case of an open or half-open interval is clearly a trivial extension since $f$ is monotone and if the domain of $f$ is open at one or both ends then $f$ extends uniquely to the closed interval. Thus, any classification involving closed intervals only can be extended to all intervals by simply adding one more label which tells whether each end is open or closed.

\section{REALIZING POTENTIAL CLASSIFICATIONS}

The above results give a complete set of invariants which tells whether a given pair of pwm function on $[0,1]$ are strongly conjugate. Given that the objects $C_{f}$ are somewhat abstract objects, there is a natural question which can be asked: Given a triple $C=\{\mathrm{sgn}, E, q\}$ which looks as though it might be a $C_{f}$ for some function $f$, how do we tell if that is actually the case? Claiming to answer such a question is of course meaningless if we use the existence of such an $f$ as our criterion. What is needed to answer this question is a criterion which is completely in the language of $\operatorname{sgn}, E$, and $q$. Most of the remainder of this paper will address this problem.

\section{LINEAR ORDERS}

Let $(A, \leq)$ be a linear ordering with associated strict linear ordering $<$.

25. Definition. If $x, y$ are elements of $A, x<y$, then $x$ and $y$ are adjacent if there is no $z$ so that $x<z<y$. A is dense if it has no two adjacent elements. $X$ is a nearest neighbor of $y$ if $x$ is adjacent to $y$. $A$ is complete if every bounded subset of $A$ has a l.u.b. and a g.l.b. The order topology on $A$ is the topology having basic open sets $\{x: x<a\},\{x: x>a\}$, and $\{x: a<x<b\}$. Recall that if $B$ is a subset of $A$, then the order topology on $(B,<)$ is not necessarily the same as the subspace topology of the order topology on $A$. We call $A$ separable or connected if its order topology has the indicated property.

Two easy facts from folklore which will be occasionally used are that if $A$ is dense, then $A$ is connected iff $A$ is complete, and that if $A$ is connected, separable, and has a greatest and least element, then $A$ is homeomorphic to the unit interval $I$.

\section{COMBINATORICS OF ITINERARIES}

In this section we examine sequences of integers, viewing them as potential itineraries of some as yet unspecified function $f$ and point $x$. While the functions will occasionally be mentioned as motivation for the definitions, we want the definitions to be combinatorial in nature and not mention the functions in these basic definitions. 
One of the main things we would like to know when is a potential itinerary is actually realized by some point. This was investigated by Guckenheimer in [G]. In what follows we do a more detailed investigation along the same lines which will be needed to nail down the conjugacy classes. Theorem 4 gives us the motivation for the following definition.

26. Definition. Let $E=\{\mathbf{e}(0), \mathbf{e}(2), \mathbf{e}(4), \ldots, \mathbf{e}(2 N)\}$ be elements of $S_{N}$ such that $e_{0}(k)=k$ for all even $k$. Let sgn be one of the two possible functions described above. Then a sequence a in $S_{N}$ is said to be E-acceptable with respect to sgn iff

(a) For any shift $\mathbf{b}$ of $\mathbf{a}$, if $b_{0}=i$ is even, then $\mathbf{b}=\mathbf{e}(i)$.

(b) For any shift $\mathbf{b}$ of $\mathbf{a}$, if $b_{0}=i$ is odd, then

$$
\begin{gathered}
S(\mathbf{e}(i-1)) \leq S(\mathbf{b}) \leq S(\mathbf{e}(i+1)) \quad \text { if } \operatorname{sgn}(i)=1, \\
S(\mathbf{e}(i-1)) \geq S(\mathbf{b}) \geq S(\mathbf{e}(i+1)) \quad \text { if } \operatorname{sgn}(i)=-1 .
\end{gathered}
$$

(c) For any shift $\mathbf{b}$ of $\mathbf{a}$ and any even $k$, if $\left|e_{1}(k)-b_{1}\right| \leq 1$ for all $i, b_{0}$ is odd, and $e_{j}(k)$ is even, then $b_{j}$ is odd.

This definition depends on the set $E$ only and is without regard to whether $E=E_{f}$ for some $f$. The set $E$ and the function sgn will usually be fixed in a given context, in which case we will write acceptable for $E$-acceptable.

A set $E$ will be called acceptable iff

(1) Every element of $E$ is $E$-acceptable.

(2) If $k$ is even, $0 \leq k<2 N$, and $S(\mathbf{e}(k))=S(\mathbf{e}(k+2))$, then $S(\mathbf{e}(k))$ is totally odd.

(3) If $k$ is odd, then

(a) If $\operatorname{sgn}(k)=1$, then $S(\mathbf{e}(k-1)) \leq S(\mathbf{e}(k+1))$, and

(b) If $\operatorname{sgn}(k)=-1$, then $S(\mathbf{e}(k-1)) \geq S(\mathbf{e}(k+1))$.

An element $\mathbf{c}(i)$ of $E$ will be called a $\max$ if $\operatorname{sgn}(i-1)=-1$ and a $\min$ if $\operatorname{sgn}(i-1)=1$.

Clearly, if $f$ is pwm, then $E_{f}$ is $E$-acceptable. We will eventually show that every acceptable $E$ is $E_{f}$ for some pwm $f$.

In the following definitions and theorems, we assume $E$ is some fixed acceptable set of sequences in $S_{N}$ for some positive integer $N$. Unless explicitly stated in the definition or theorem, we will not be assuming that $E$ is an $E_{f}$ for some $f$, since we have not yet shown that an acceptable $E$ has that property. However, it is useful to think about what happens with an $E_{f}$ when reading these theorems in order to get some motivation as to the reasons for the definitions and theorems.

27. Proposition. If a is acceptable then so is every shift of $\mathbf{a}$, and, if $\mathbf{a}$ is unacceptable then so is every preshift of $\mathbf{a}$.

Proof. Obvious from definition.

28. Definition. If $E$ is acceptable, then define $A_{E}=\{\mathbf{a}: \mathbf{a}$ is acceptable $\}$. Note that $A_{E}$ contains $E$ and by Definition $26(\mathrm{a})$ is linearly ordered by $<$. Give $A_{E}$ the order topology. 
29. Definition. Extend the definition of acceptable to finite sequences by calling a finite sequence $\mathbf{a}$ acceptable iff $\mathbf{a b}$ is acceptable for some infinite $\mathbf{b}$. However, we do not put such finite sequences in $A_{E}$.

30. Theorem. $A_{E}$ is complete, separable, and has a first and last element.

Proof. $\mathbf{e}(0)$ and $\mathbf{e}(2 N)$ are clearly the first and last elements, respectively. For each finite acceptable $\mathbf{a}$ pick $\mathbf{b}_{a}$ such that $\mathbf{a b}_{a}$ is acceptable. Then $\left\{\mathbf{a b}_{a}: a\right.$ finite and acceptable $\}$ is a countable dense subset of $A_{E}$. Since $A_{E}$ is separable and has a first and last element, to show completeness it suffices to show that every monotone sequence in $A_{E}$ has a limit in $A_{E}$. Suppose WLOG a $(0)<$ $\mathbf{a}(1)<\cdots<\mathbf{a}(n)<\cdots$. It is easy to see (by taking a subsequence if necessary) that for all $i<j, a_{i}(j)=a_{i}(1)$. Let $\mathbf{a}=\left(a_{0}(0), a_{1}(i), a_{2}(2), \ldots, a_{n}(n), \ldots\right)$. Then $\mathbf{a}$ is clearly the l.u.b. of the sequence and it is routine to check that $\mathbf{a}$ is acceptable.

31. Lemma. If $\mathbf{a}$ is acceptable and $\mathbf{a}=\mathbf{e}(0)$, then there is a totally odd acceptable $\mathbf{b} \geq \mathbf{a}$ such that $\left|b_{i}-a_{i}\right| \leq 1$ for all $i$. If $\mathbf{a}$ is acceptable and $\mathbf{a}=\mathbf{e}(2 N)$, then there is a totally odd acceptable $\mathbf{b} \geq \mathbf{a}$ such that $\left|b_{i}-a_{i}\right| \leq 1$ for all $i$.

Proof. We prove only the first statement, the second being symmetric. Define $b_{i}$ by induction on $i$. Assume $b_{j}$ has been defined for all $j<i$. If $a_{i}$ is odd we let $b_{i}=a_{i}$. If $a_{i}$ is even, we let $b_{i}=a_{i}-1$ if $\operatorname{sgn}\left(b_{i}\right)$ is odd for an even number of $j<i$, and we let $b_{i}=a_{i}+1$ otherwise. It is now routine to check that $\mathbf{b}$ is acceptable.

32. Lemma. If $\mathbf{a}$ is acceptable, $i \geq 0$, and $a_{i+1}=0$, then $a_{i}$ is even, $\operatorname{sgn}\left(a_{i}-1\right)=-1$ and $\operatorname{sgn}\left(a_{i}+1\right)=1$. If $\mathbf{a}$ is acceptable, $i \geq 0$, and $a_{i+1}=2 N$, then $\mathbf{a}_{i}$ is even, $\operatorname{sgn}\left(a_{i}-1\right)=1$ and $\operatorname{sgn}\left(a_{i}+1\right)=-1$.

Proof. We prove only the first sentence, the other one being symmetric. If $a_{i+1}=0$, then $\mathbf{a}=\mathbf{b e}(0)$ where $\mathbf{b}=\left(a_{0}, a_{1}, a_{2}, \ldots, a_{i}\right)$, by Definition 26(a). If $a_{i}$ is odd, then Definition 26(b) applied to $\mathbf{a}_{i} \mathbf{e}(0)=S^{k}(\mathbf{a})$ implies that $S(\mathbf{e}(i-1))=S(\mathbf{e}(i+1))=\mathbf{e}(0)$, regardless of whether $\operatorname{sgn}\left(\mathbf{a}_{i}\right)$ is -1 or 1 . But then $S(\mathbf{e}(i-1))$ is not totally odd, violating the definition of $E$-acceptable. Thus $a_{i}$ must be even. Let $\mathbf{b}$ be totally odd and acceptable such that $\left|b_{i+1}-e_{i}(0)\right| \leq 1$ for all $i,\left|b_{0}-a_{i}\right| \leq 1$, by using the previous lemma. Now, since $S\left(a_{i} \mathbf{e}(0)\right)=$ $\mathbf{e}(0)<S(\mathbf{b})$, since $\mathbf{b}$ is totally odd, it is easy to see that Definitions $26(\mathrm{~b})$ gives the rest of the lemma.

33. Lemma. If $\mathbf{a}$ and $\mathbf{b}$ are acceptable, at least one of which is totally odd and $\left|a_{i}-b_{i}\right| \leq 1$ for all $i$, then there is no acceptable $\mathbf{c}$ such that $\mathbf{c}$ is between a and $\mathbf{b}$, i.e. $\mathbf{a}$ and $\mathbf{b}$ are adjacent.

Proof. By symmetry, assume $\mathbf{a}$ is not totally odd with $\mathbf{a}<\mathbf{b}$. Then there cannot be a $\mathbf{c}$ so that $\mathbf{a}<\mathbf{c}<\mathbf{b}$, for suppose such a $\mathbf{c}$ exists. Then an easy induction gives that $a_{i} \leq c_{i} \leq b_{i}$ if $\operatorname{sgn}\left(b_{j}\right)=-1$ for an odd number of $j<i$, and $a_{i} \leq c_{i} \leq b_{i}$ if $\operatorname{sgn}\left(b_{j}\right)=-1$ for an odd number of $j<i$. But $c_{i}$ must be even for at least one $i$ and $\left|c_{i}-a_{i}\right| \leq 1$ for all $i$, which contradicts either 
(a) or (c) of Definition 26, depending on whether the first even entry of $\mathbf{c}$ is before or after the first even entry of $\mathbf{a}$. Thus no such $\mathbf{c}$ exists, and $\mathbf{a}$ and $\mathbf{b}$ are adjacent.

34. Corollary. Any element of $A_{E}$ which is not totally odd is an isolated point of $A_{E}$.

35. Lemma. The shift function satisfies the intermediate-value theorem in $A_{E}$, i.e. if $\mathbf{a}<\mathbf{b}$ and $\mathbf{d}$ is between $S(\mathbf{a})$ and $S(\mathbf{b}), \mathbf{a}, \mathbf{b}, \mathbf{d}$ all acceptable, then there is an acceptable $\mathbf{c}$ such that $\mathbf{a}<\mathbf{c}<\mathbf{b}$ and $S(\mathbf{c})=\mathbf{d}$.

Proof. We may assume that $\left|b_{0}-a_{0}\right| \leq 1$, since if not it is easy to find acceptable $\mathbf{a}^{\prime}, \mathbf{b}^{\prime}$ such that $\mathbf{a} \leq \mathbf{a}^{\prime}<\mathbf{b}^{\prime} \leq \mathbf{b},\left|b_{0}^{\prime}-a_{0}^{\prime}\right| \leq 1$, and $\mathbf{d}$ is between $S\left(\mathbf{a}^{\prime}\right)$ and $S\left(\mathbf{b}^{\prime}\right)$, and then proving for $\mathbf{a}^{\prime}, \mathbf{b}^{\prime}$ gives the lemma for $\mathbf{a}, \mathbf{b}$. Thus we may divide into two cases:

Case 1. $a_{0}+1=b_{0}$. By symmetry, we may assume $b_{0}=i$ is odd. Let $\mathbf{c}=(i) \mathbf{d}$. Then it is easy to check that $\mathbf{c}$ is acceptable and as desired (part (b) of Definition 26 is all that needs to be checked, and shifts of $\mathbf{c}$ need not be checked, since $\mathbf{d}$ is already known to be acceptable).

Case 2. $a_{0}=b_{0}$. Then since $\mathbf{a}<\mathbf{b}, a_{0}=b_{0}=i$ must be odd, and we can now let $\mathbf{c}=(i) \mathbf{d}$ and argue as in Case 1 .

36. Lemma. Suppose $\mathbf{a}<\mathbf{b}$ are acceptable and totally odd. Then there is an acceptable $\mathbf{c}$ such that $\mathbf{a}<\mathbf{c}<\mathbf{b}$.

Proof. Let $k$ be least such that $a_{k}=b_{k}$. Then $S^{k}(\mathbf{a})<\mathbf{e}(i)<S^{k}(\mathbf{b})$, where $i$ is even and between $a_{k}$ and $b_{k}$. Applying Lemma $35 k$ times now gives $\mathbf{c}$ such that $\mathbf{a}<\mathbf{c}<\mathbf{b}$ and $S^{k}(\mathbf{c})=\mathbf{e}(i)$.

37. Lemma. $S$ is a continuous function on $A_{E}$.

Proof. Let $\left(x_{n}\right)$ be a sequence, all acceptable, which converges to $x$. WLOG and by symmetry, we may assume that the sequence is a strictly increasing sequence, and by Corollary 34 we know that $x$ must be totally odd. By Lemmas 31 and 33, we may assume (by replacing each nontotally odd $x_{n}$ by the next largest element of $\left.A_{E}\right)$ that each $x_{n}$ is totally odd. It is now easy to see that the sequence $\left(S\left(x_{n}\right)\right)$ converges to $S(x)$.

\section{OPTIONAL AND MANDATORY SEQUENCES}

38. Definition. Given a in $A_{E}$, a is called optional iff every acceptable b\#a is both totally odd and has exactly one nearest neighbor in $A_{E}$. If a is not optional, then a will be called mandatory, i.e. if there exists an acceptable b\#a which is either not totally odd or has either 0 or 2 nearest neighbors in $A_{E}$.

Two examples which help to motivate this definition are given in the illustration below.

Note that $E=E_{f}=E_{g}=\left\{0^{*},(2,4)\left(0^{*}\right),(4)\left(0^{*}\right)\right\}$. It is an easy exercise to check that $\left(1^{*}\right)$ is optional $A_{E}$. The function $f$ has points $x$ such that 

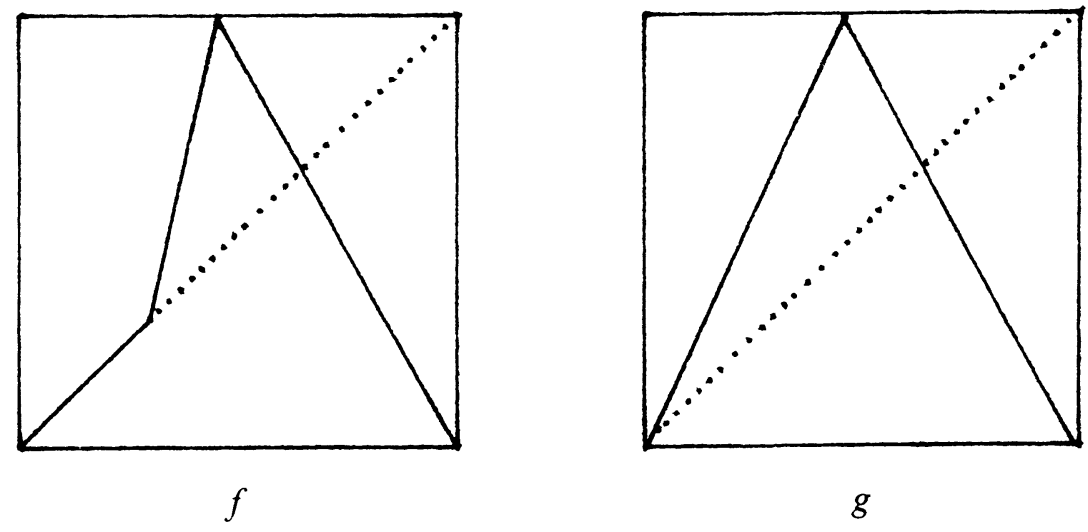

FIGURE 2

$\mathbf{a}_{f}(x)=\left(1^{*}\right)$ but $g$ does not, so $\left(1^{*}\right)$ is an itinerary which does not have to be realized by a function $h$ such that $E_{h}=E$. On the other hand, $\left(3^{*}\right)$ is mandatory (it has no nearest neighbor), and it is easy to see that every pwm $h$ such that $E_{h}=E$ must have a point $x$ (i.e. the fixed point in the decreasing interval) such that $\mathbf{a}_{h}(x)=\left(3^{*}\right)$.

We define the following two subsets of $A_{E}$ :

$$
0_{E}=\{\mathbf{a}: \mathbf{a} \text { is optional }\}, \quad M_{E}=\{\mathbf{a}: \mathbf{a} \text { is mandatory }\} .
$$

Give $M_{E}$ the order topology, and note that in most cases this is not the same as the subspace topology viewing $M_{E}$ as a subset of $A_{E}$.

39. Lemma. If $\mathbf{a}$ and $\mathbf{b}$ are optional, $\mathbf{a}<\mathbf{b}$, then there exists $\mathbf{c}$ in $A_{E}$ such that $\mathbf{a}<\mathbf{c}<\mathbf{b}$.

Proof. Immediate from the definition and Lemma 36.

40. Proposition. If $\mathbf{a}$ is optional, then so is every b\#a. If a is mandatory, then so is every b\#a.

41. Corollary. $\left(M_{E},<\right)$ is a complete, separable, linearly-ordered topological space with first and last element.

Proof. We only need to prove completeness, the rest being a trivial consequence of the same result for $A_{E}$. Let $X$ be a bounded subset of $M_{E}$. Then $X$ has a supremum $\mathbf{b}$ in $A_{E}$, since $A_{E}$ is complete. If $\mathbf{b}$ is mandatory then we are done. If $\mathbf{b}$ is optional then $\mathbf{b}$ has exactly one nearest neighbor in $A_{E}$, say $\mathbf{c}$, which must be mandatory by Lemma 39 . Then c must be the supremum of $X$ in $M_{E}$.

42. Theorem. The shift map $S$ is continuous $M_{E}$.

Proof. Let $\left(x_{n}\right)$ be an increasing sequence from $M_{E}$, and let $x$ be its supremum. We need to show that $S(x)$ is the supremum of $\left(S\left(x_{n}\right)\right)$ in $M_{E}$, but that is a trivial consequence of the fact that $S$ is continuous on $A_{E}$ and $x$ and $S(x)$ must both be mandatory. 


\section{SEQUENCES AND FUNCTIONS}

We now want to prove some theorems which give justification to the terms acceptable, mandatory, and optional. For purposes of this section we will be considering a fixed pwm $f$, with $E=E_{f}$, with terms such as acceptable always with respect to this fixed $f$ and $E$. Recall from above that $I_{a}=\left\{x: \mathbf{a}_{f}(x)=\right.$ a).

43. Theorem. For all finite a, $I_{a}$ is nonempty iff a is acceptable.

Proof. $(\Rightarrow)$ obvious from definitions.

$(\Leftarrow)$ By induction on the length of $\mathbf{a}$. It is obvious if a has length 1 . Suppose the theorem is true for all $\mathbf{b}$ of length $n$ and let a have length $n+1$, say $\mathbf{a}=(i) \mathbf{b}$, a acceptable. If $i$ is even, then we are done since then $\mathbf{a}=\mathbf{e}(i) \mid(n+1)$ and $I_{a}=I_{i}$, so assume $i$ is odd. We consider only the case $\operatorname{sgn}(i)=1$, the case $\operatorname{sgn}(i)=-1$ being virtually identical.

Claim. $\left[f\left(t_{i-1}\right), f\left(t_{i+1}\right)\right] \cap I_{b}$ is nonempty.

Proof of claim. By the induction hypothesis, there is a point $x$ in $I_{b}$. If $f\left(t_{i-1}\right) \leq x \leq f\left(t_{i+1}\right)$ we are done, so assume, for example, that $f\left(t_{i+1}\right)<x$ (the case $x<f\left(t_{i-1}\right)$ is similar). Then $\mathbf{a}_{f}\left(f\left(t_{i+1}\right)\right) \leq \mathbf{a}_{f}(x)$. Letting $\mathbf{c}=\mathbf{a}_{f}(x)$, this inequality can be restated as $S(\mathbf{e}(i+1)) \leq \mathbf{c}$. By the acceptability of a, there is an infinite acceptable $\mathbf{d}$ such that $\mathbf{a}$ is an initial segment of $\mathbf{d}$. By acceptability of $\mathbf{d}, S(\mathbf{e}(i-1)) \leq S(\mathbf{d}) \leq S(\mathbf{e}(i+1))$. But this gives $S(d) \leq S(\mathbf{e}(i+1)) \leq \mathbf{c}$. Now $S(\mathbf{d})|n=\mathbf{b}=\mathbf{c}| n$, so $S(\mathbf{e}(i+1))$ must have $\mathbf{b}$ as its first $n$ coordinates, putting $f\left(t_{i+1}\right)$ in $I_{b}$, a contradiction. This finishes the proof of the claim.

If $I_{b}$ intersects $\left(f\left(t_{i-1}\right), f\left(t_{i+1}\right)\right)$ then we are done, so the only possible problem that remains is if $\left[f\left(t_{i-1}\right), f\left(t_{i+1}\right)\right]$ intersects $I_{b}$ only at an endpoint, say at $f\left(t_{i+1}\right)$, so $I_{b}$ cannot be an open interval, and by Proposition 7 , b cannot be totally odd, and there must be a $j<n$ so that $\mathbf{b}_{j}=\mathbf{a}_{j+1}=k$ for some even $k$. But then $\mathbf{a}_{n}=\mathbf{e}_{n}(i+1)$ for all $n$ and $\left|\mathbf{a}_{0}-\mathbf{e}(i+1)\right|=1$, contradicting part (c) in the definition of acceptable (Definition 26).

44. Theorem. Let a be mandatory. Then there is an $x$ is I such that $\mathbf{a}_{f}(x)=$ a. In other words, mandatory sequences must be realized as the itinerary of some point.

Proof. For each $j$ let $J_{j}=I_{a \mid j}$. Then $J_{j}$ is nonempty and $J_{j+1}$ is a subset of $J_{j}$ for all $j$. If $\bigcap\left\{J_{j}: j \geq 0\right\}$ is nonempty, we are done, so assume $\bigcap\left\{J_{j}: j \geq 0\right\}$ is empty. Then $J_{j}$ must be an open interval for every $j$, and there must be a final segment of $j$ 's such that $J_{j}$ has the same endpoint on one end, say by symmetry there is an $x$ such that $x$ is the upper endpoint of $J_{j}$ for $j>n$. Now, a must be totally odd since the $J_{j}$ 's are open. Let $\mathbf{b}=\mathbf{a}_{f}(x)$, assuming that $\mathbf{a}=\mathbf{b}$ let $k$ be large enough so that $\mathbf{a}|k=\mathbf{b}| k$. Then we must have $x$ as an upper endpoint of $J_{k}$, with $I_{b \mid k}=\{x\}$. There can be no $\mathbf{d}$ in $A_{E}$ such that $\mathbf{a}<\mathbf{d}<\mathbf{b}$ since if we let $m$ be large enough so that $\mathbf{a} \mid m$, and 
b $\mid m$ are all distinct we will have $I_{a \mid m}<I_{d \mid m}<I_{b \mid m}$, a contradiction. Thus $\mathbf{a}$ and $\mathbf{b}$ are nearest neighbors, and $\mathbf{a}$ cannot have another nearest neighbor $\mathbf{c}$, say $\mathbf{c}<\mathbf{a}<\mathbf{b}$, since then we would have $I_{a \mid i}=(y, x)$ for all sufficiently large $i$, where $y$ is such that $\mathbf{a}_{f}(y)=\mathbf{c}$ (which must exist since $c$ could not be totally odd). Thus $\mathbf{a}$ is totally odd and has exactly one nearest neighbor. If $\mathbf{b}$ is a preshift of $\mathbf{a}$, say $\mathbf{a}=S^{i}(\mathbf{b})$, then there cannot be a $z$ in $I$ with $\mathbf{a}_{f}(z)=\mathbf{b}$ since we would then have $\mathbf{a}_{f}\left(f^{i}(z)\right)=\mathbf{a}$. Thus the same argument as given above for a applies to any preshift of a . The same argument can be applied to any shift of $\mathbf{a}$, and thus to any $\mathbf{b} \# \mathbf{a}$, and thus $\mathbf{a}$ is optional, a contradiction.

Recall the definition of a weakly expanding function $f$ (i.e. $\mathbf{a}_{f}$ is one-toone) above (Proposition 10). A corresponding definition can be given in terms of $M_{E}$ :

45. Definition. $E$ is called weakly expanding iff $E$ is $E$-acceptable and $M_{E}$ is a dense linear ordering.

46. Theorem. Let $f$ by pwm. If $f$ is weakly expanding then so is $E_{f}$. Conversely, if $E$ is weakly expanding, then there is a weakly expanding pwm function $f$ on $I$ such that $E_{f}=E$.

Proof. $(\Rightarrow)$ Suppose $f$ is weakly expanding and let $E=E_{f}$. Let $\mathbf{a}, \mathbf{b}$ be in $M_{E}$. Then there are $x<y$ in $I$ so that $\mathbf{a}_{f}(x)=\mathbf{a}$ and $\mathbf{a}_{f}(y)=b$. Let $z, w, v$ be such that $x<z<w<v<y$. Then since $f$ is weakly expanding, $\mathbf{a}=\mathbf{a}_{f}(x)<\mathbf{a}_{f}(z)<\mathbf{a}_{f}(w)<\mathbf{a}_{f}(v)<\mathbf{a}_{f}(y)=\mathbf{b}$. Then either $\mathbf{a}_{f}(w)$ is in $M_{E}$ or is adjacent to an element of $M_{E}$. In either case there must be an element of $M_{E}$ strictly between a and $\mathbf{b}$, so $M_{E}$ is dense.

$(\Leftarrow) M_{E}$ is dense, separable, and complete and is thus homeomorphic to $I$ by an order-preserving homeomorphism $h$ from $M_{E}$ to $I$. Let $f=h S h^{-1}$ and it is then easy to check that $f$ is pwm with $E_{f}=E$.

47. Theorem. If $f$ is weakly expanding, then $\mathbf{a}_{f}(x)$ is mandatory for every $x$ in $I$.

Proof. It is easy to check that in this case $\mathbf{a}_{f}(x)$ cannot have a nearest neighbor in $A_{E}$.

The following theorem shows that if we restrict ourselves to the weakly expanding pwm functions, the our sets $E_{f}$ in fact give us a complete set of invariants which classify the (strong) conjugacy classes of all such functions.

\section{OPTIONAL SEQUENCES}

In this section we prove a few results regarding optional sequences which will finally give complete justification for the terms acceptable, mandatory, and optional.

48. Theorem. Let $f$ be pwm on $I$. Then there is a pwm function $g$ on $I$ such that $E_{f}=E_{g}$ and $\mathbf{a}_{g}(x)$ is mandatory for every $x$ in $I$. 
Proof. Let $J$ be the set of all $x$ in $I$ such that $\mathbf{a}_{f}(x)$ is mandatory, i.e., $J$ is $I$ with all $I_{a}$ thrown out for optional a. Every interval which is thrown out is half-open-half-closed. Furthermore, the endpoint at the open end of each such interval must have a mandatory itinerary and will thus never get thrown out. Thus, it is easy to check that $J$ (as a linear ordering) is separable, complete, and has first and last element. In addition, no critical point was thrown out and both $J$ and $I-J$ are closed under the function $f$. Thus, $f \mid J$ is continuous on $J$ with respect to the order topology on $J$, so let $h$ be an order-preserving homeomorphism of $J$ (with order topology) onto $I$ and let $g=h f h^{-1}$. It is now easy to see that $g$ is as desired.

49. Theorem. Let $f$ be pwm on $I, E=E_{f}$, and let a be optional. Then there is a function $g$ on $I$ such that $E_{f}=E_{g}$ and there is an $x$ in $I$ such that $\mathbf{a}_{g}(x)=\mathbf{a}$.

Proof. WLOG assume there is no $x$ such that $\mathbf{a}_{f}(x)=\mathbf{a}$. Then since $a$ is optional, a has a nearest neighbor $\mathbf{b}$ which cannot be totally odd, so there is a unique point $z$ such that $\mathbf{a}_{f}(z)=\mathbf{b}$. Let $X$ be the smallest set containing $z$ and closed under images and preimages (with respect to $f$ ). Replace each point of $X$ by a closed interval containing more than one point. Since $X$ is countable it is easy to arrange these added intervals so that the total length is still finite, and the result is homeomorphic to $I$. The function $f$ can now (by being a little careful at the critical points) be extended to a pwm $g$ such that $E_{g}=E_{f}$, and there will be points in the interval corresponding to a which have itinerary a .

50. Theorem. Let $E$ be E-acceptable. Then there is a pwm function $f$ such that $E_{f}=E$ and for every acceptable a there is an $x$ such that $\mathbf{a}_{f}(x)=\mathbf{a}$.

Proof. Embed $M_{E}$ in $I$ by an order-preserving map $h$ such that $h(\mathbf{c}(0))=0$ and $h(\mathbf{c}(2 N))=1$. If $J=h\left(M_{E}\right)$, let $g$ be the function on $J$ defined by $g=h S h^{-1}$. Let $t_{k}=h(\mathbf{c}(k))$ for even $k$. Let $X$ be the smallest set containing $\left\{t_{k}: k\right.$ even $\}$ and closed under images and inverse images with respect to $g$, and replace each element of $X$ with a nontrivial closed interval as in Theorem 49. Then $g \mid J-X$ can be extended to a function $g^{\prime}$ such that $E_{g}=E$. Since $O_{E}$ is countable, Theorem 49 can now be applied at most countably many times to get the desired $f$.

51. Definition. If $G_{k}$ is periodic then $\mathbf{d}(k)$ is the least periodic element of $[\mathbf{c}(k)]$ (with respect to the ordering $<$. Note that there are only finitely many possibilities, so the least element exists.). If $G_{k}$ is aperiodic, then $\mathbf{d}(k)$ is the unique sequence which is a shift of every element of $G_{k}$, but no preshift of $\mathbf{d}(k)$ is a shift of every element of $G_{k}$. If $\mathbf{d}(k)$ is totally odd then the relations $={ }^{*}$ and $<{ }^{*}$ on $G_{k}$ are defined by:

$\mathbf{a}={ }^{*} \mathbf{b}$ iff $\mathbf{a}$ is a shift or preshift of $b$, and

$\mathbf{a}<^{*} \mathbf{b}$ iff $\mathbf{a}=\mathbf{a}^{\prime} \mathbf{d}(k), \mathbf{b}=\mathbf{b}^{\prime} \mathbf{d}(k), \mathbf{a}^{\prime}=\left(a_{0}, a_{1}, \ldots, a_{m}\right), \mathbf{b}^{\prime}=\left(b_{0}, b_{1}\right.$, $\left.\ldots, b_{n}\right), i$ and $j$ are the smallest integers such that $m-i=n-j$ and $a_{1}=b_{j}$, 
$\left|a_{i-1}-b_{j-1}\right|=1$, and either

(1) $a_{i-1}$ is an even min and $\left(b_{j}, \ldots, b_{n}\right)$ is even, or

(2) $b_{j-1}$ is an even max and $\left(a_{i}, \ldots, a_{m}\right)$ is odd.

We define $\mathbf{a} \leq{ }^{*} \mathbf{b}$ iff either $\mathbf{a}<{ }^{*} \mathbf{b}$ or $\mathbf{a}={ }^{*} \mathbf{b}$. Then $\leq^{*}$ is a transitive reflexive ordering. It will often by the case that $\mathbf{a}$ and $\mathbf{b}$ are not related with respect to the $\leq^{*}$ ordering.

52. Definition. Let $H$ be the set of all \#-equivalence classes. For each acceptable sequence a we define $G$ [a] to be $G_{k}$ if $\mathbf{a}=\mathbf{c}(k)$ for some $k$ and $G[\mathbf{a}]$ is the empty set otherwise. Given a in $A_{E}$ and $Q(f, F)$ in $P$, and a function $r$ from $G[\mathbf{a}]$ onto $F$, the triple ([a], $Q(f, F), r)$ will be called monotone iff the following conditions hold:

(1) If $\mathbf{c}(i)={ }^{*} \mathbf{c}(j)$ then $r(\mathbf{c}(i))=r(\mathbf{c}(j))$.

(2) If $\mathbf{c}(i) \leq^{*} \mathbf{c}(j)$ then $r(\mathbf{c}(i))<r(\mathbf{c}(j))$.

(3) If $f$ has empty domain, then $a$ is optional.

(4) If $f$ has nonempty domain, then $a$ has an immediate predecessor in $A_{E}$ iff the domain of $f$ has no least element.

(5) If $f$ has nonempty domain, then $a$ has an immediate successor in $A_{E}$ iff the domain of $f$ has no greatest element.

Note that in all but finitely many cases the function $r$ will have empty domain, and that if $\mathbf{a}$ is optional then the domain of $f$ is either the empty set or a half open interval.

53. Definition. A classification will be any triple $(\operatorname{sgn}, E, q)$ satisfying the following properties:

(a) $E$ is an $E$-acceptable subset of $S_{N}$ for some $N$ (with respect to sgn).

(b) sgn is one of the two possible sign functions.

(c) $q$ is a function with domain $H$ such that for every element [a] of $H$, $q[\mathbf{a}]$ is a pair $(Q(f, F), r)$ such that

(1) ([a], $Q(f, F), r)$ is monotone.

(2) If [a] is aperiodic then $f$ is an identity function

(3) If [a] has no totally odd element, then $Q(f, F)=Q_{1}^{*}$.

(4) $Q(f, F)$ is $Q_{0}$ or $Q_{1}$ for all but countably many [a] .

54. Theorem. Every classification is realized, i.e., if $C$ is a classification, then there is a pwm function $f$ such that $C_{f}=C$.

Proof. Given a classification $C=(\operatorname{sgn}, E, q)$, for each acceptable a we define an interval $I_{a}$ according to the following cases.

Case 1. a not totally odd. Let $I_{a}$ be a singleton.

Case 2. a is totally odd. $I_{a}$ is an interval $(x, y),(x, y],[x, y)$, or $[x, y]$. We decide among these four cases by letting $I_{a}$ be closed at the lower end iff $a$ has no immediate predecessor and closed at the upper end iff $a$ has no immediate successor. The value of $q[\mathbf{a}]$ is used to decide whether $I_{a}$ is trivial (i.e. a point or empty) or has interior. We now get a linear ordering by taking 
the union of all the $I_{a}$ 's and ordering elements of different $I_{a}$ 's according to the <-ordering on $A_{E}$. Since any two adjacent elements of this ordering must have one totally odd and the other not totally odd, it is easy to see by the cases above that this ordering is connected. In addition, only countably many $q$ [a]'s can be nontrivial, so we can assume that $I$ is the union of all the $I_{a}$ 's. We now define $f$ on $I_{a}$ for each a much as in the previous theorem. For example, if $\mathbf{a}$ is totally odd and periodic with least period $k$ (the most difficult case), let $q[\mathbf{a}]=((g, F), r)$ and WLOG assume $\mathbf{a}<S^{i}(\mathbf{a})$ for all $i, 0<i<k$. Pick points $F^{\prime}$ in $I_{a}$ and a function $g^{\prime}$ on $I_{a}$ so that $Q(g, F)=Q\left(g^{\prime}, F^{\prime}\right)$. This can be easily used to define a function $f$ on $I_{b}$ for each shift $b$ of $a$ so that $f^{k} \mid I_{a}=g^{\prime}$, since $a$ has least period $k$. We then extend $f$ to $I_{b}$ for the preshifts $\mathbf{b}$ of $\mathbf{a}$, using the function $r$ to make sure that the points corresponding to any $c(j)$ map in the correct way to the finite set $F$. The relations $<^{*}$ and $=^{*}$ are guarantee that this can be done in a continuous way. If [a] is aperiodic, the argument is the same except that if $\mathbf{b}$ is a shift of $\mathbf{a}$, then the intervals $I_{a}$ and $I_{b}$ never intersect, so only the order of the points $F$ need to be considered. This defines the function $f$ on the entire interval $I$, and it is easy to see that $f$ is as desired.

The above theorem completes the "realization" part of the classification of the pwm functions on $I$. This classification system was motivated by the onedimensional dynamical system defined by the map $f$ and gives substantial information about that dynamical system. The set $E_{f}$ gives the history of the turning points of $f$, the most important points of $f$ which tell most but not always all of the story. The function $q_{f}$ gives us information about the attractors of $f$. If [a] is periodic then $q_{f}$ [a] tells us about the attracting periodic points (if any) having "type" a. If [a] is aperiodic then $q_{f}[\mathbf{a}]$ tells us about the possible attractors of $f$ which are Cantor sets.

There is one problem which provided some of the motivation for the effort to get a classification of the piecewise monotone functions, and that is the related but much more difficult job deciding when the inverse limit spaces defined by two different bonding maps on $I$ are homeomorphic. If we let $X_{f}$ be the inverse limit space defined using $f$ as a bonding map, where $f$ is a function on $I$, then it is clear that if $f$ and $g$ are conjugate, then $X_{f}$ and $X_{g}$ are homeomorphic. The converse is obviously not true since $f$ and $f^{2}$ give the same inverse limit. However, we can still ask whether or not the classifications $C_{f}$ and $C_{g}$ of two pwm functions $f$ and $g$ can give us any information about whether or not their inverse limits are homeomorphic. Although no progress has been made on this problem (at least by this author) there is one interesting fact that suggests that this classification might be useful in dealing with this problem. Let $f$ be pwm and weakly expanding, and consider a sequence $\mathbf{a}=\left\{\ldots a_{-2}, a_{-1}, a_{0}, a_{1}, a_{2}, \ldots\right\}$ which is infinite at both ends. Call such a sequence a mandatory iff every final segment of a is mandatory in $E_{f}$. Then it is easy to see that $X_{f}$ and $\{\mathbf{a}: \mathbf{a}$ is mandatory $\}$ have a natural one-to-one cor- 
respondence, something which might be of use in deciding the homeomorphism class of $X_{f}$.

\section{BIBLIOGRAPHY}

[BC] Louis Block and Ethan M. Coven, Topological conjugacy and transitivity for a class of piecewise monotone maps of the interval, preprint.

[CE] Pierre Collet and Jean-Pierre Eckmann, Iterated maps on the interval as dynamical systems, Birkhäuser, 1980.

[G] John Guckenheimer, Bifurcations of dynamical systems, Dynamical Systems, C.I.M.E. Lectures, Progress in Math., Vol. 8, Birkhäuser, 1980, pp. 115-231.

[MSS] M. Metropolis, M. L. Stein, and P. R. Stein, On finite limit sets for transformations of the unit interval, J. Combin. Theory 15 (1973), 25-44.

[MT] J. Milnor and P. Thurston, On iterated maps of the interval. I, II, Princeton Univ. Press, 1977.

Department of Mathematics, Auburn University, Auburn, Alabama 36849 